\title{
Carcinoma de Tireóide na Infância e Adolescência
}

\section{RESUMO}

Aproximadamente $10 \%$ das neoplasias de tireóide aparecem abaixo dos 21 anos e representam $3 \%$ das neoplasias da infância, com predomínio em meninas (2F:1M). São papilíferos em $90 \%$ casos e mais freqüentemente bilaterais, multifocais e de maior dimensão. Invasão de cápsula e metástases ganglionar ou pulmonar são mais freqüentes que no adulto. $O$ efeito carcinogênico da radiação é especialmente importante na criança pré-púbere. Em $5 \%$ dos casos de PTC existe história familial. Genes como o RET/PTC, RAS e BRAF estão habitualmente envolvidos na gênese do processo. A apresentação clínica pode ser nódulo cervical ( $90 \%$ casos) sem que represente um fator de pior prognóstico. O US e a PAAF são procedimentos diagnósticos valiosos. O tratamento deve ser cirúrgico (tireoidectomia total com esvaziamento ganglionar) seguido de $\mathrm{PCl}$ e radioterapia ablativa. A substituição com L-tiroxina deve objetivar a supressão crônica do TSH. O prognóstico ao longo prazo é melhor que no adulto. A quantificação da Tg plasmática também é útil na detecção da doença residual. (Arq Bras Endocrinol Metab 2007;51/5:763-768)

Descritores: Câncer de tireóide; Crianças e adolescentes; Metástases; Ca papilífero

\section{ABSTRACT}

\section{Thyroid Carcinoma in Children and Adolescents.}

Approximately $10 \%$ of thyroid cancers are present in patients less than 21 years of age, representing $3 \%$ of all cancers of children and adolescents, with predominance in females 2:1 in relation to males. Thyroid cancers in this age group are usually papillary $(90 \%)$, bilateral, multifocal and bigger in size compared to adults. Capsule invasion and lymphatic and pulmonary metastases are more frequent in children. Radiation sensitivity seems to represent an important factor in prepubertal patients. Familial history is reported in $5 \%$ of the cases. Genes such as RET/PTC, RAS and BRAF are usually involved in thyroid carcinogenesis in this age group. Cervical adenomegaly is a common clinical presentation, but does not represent a poor prognostic factor in children. Ultrasound and fine needle aspiration biopsy are valuable diagnostic procedures. Surgery is the preferred treatment including thyroidectomy and ganglionary excision, followed by ablative radioiodine therapy. L-thyroxine replacement with suppressive dosage should be employed targeting chronic TSH suppression. Long-term prognosis is usually better in children when compared with adults. Plasma thyroglobulin measurement is also useful to detect residual thyroid cancer disease. (Arq Bras Endocrinol Metab 2007; 51/5:763-768)

Keywords: Thyroid cancer; Children and adolescents; Metastasis; Papillary cancer

\footnotetext{
INCIDÊNCIA DA DOENÇA NODULAR da tireóide na criança entre 9 e 18 A anos de idade varia de $0,2 \%$ a 1,8\% (1). Apenas $10 \%$ dos cânceres de tireóide se desenvolvem na faixa etária abaixo de 21 anos (2). Nas crianças, a maior parte dos carcinomas de tireóide é derivada das células foliculares
}

\section{revisão}

\author{
OSMAR MONTE \\ LUIS EdUardo P. Calliari \\ CRISTIANE KOCHI \\ NILSA MARIA SCALISSE \\ MARILIA MARONE \\ Carlos alberto longul
}

\author{
Unidade Endocrinologia \\ Pediátrica do Departamento \\ de Pediatria e Puericultura (OM, \\ LEPC, CK \& CAL) e Disciplina \\ Endocrinologia do \\ Departamento da Clínica \\ Médica (OM, NMS \& MM),, \\ Irmandade da Santa Casa de \\ Misericórdia de São Paulo/ \\ Faculdade de Ciências \\ Médicas da Santa Casa de \\ São Paulo, SP.
}


$(3,4)$, sendo o carcinoma derivado das células parafoliculares menos freqüiente. $\mathrm{O}$ câncer de tireóide representa $6 \%$ de todos os tumores na região cervical $(5,6)$ e corresponde a, aproximadamente, $1,5 \%$ a $3 \%$ de todos os cânceres da infância na América do Norte e Europa, com incidência anual de 0,5 a 1 caso por milhão de crianças $(7,8)$. É pouco freqüente antes dos 10 anos (9), sendo as meninas mais afetadas que os meninos na proporção de 2 a 2,5:1 (10). Em nossa casuística, detectamos 15 pacientes do sexo feminino e 5 do sexo masculino, com idade média ao diagnóstico de 12 anos (12).

\section{PATOLOGIA}

$\mathrm{Na}$ criança e no adolescente, quase todos os carcinomas de tireóide são papilíferos (PTC) (18/20 em nossa casuística), sendo o folicular (1/20) e o medular (1/20) menos freqüentes $(11,12)$. Em crianças mais novas, a forma esclerosante difusa e a variante folicular do PTC são mais freqüentes (13). Quando comparado ao PTC do adulto, durante a infância o tumor é mais freqüentemente bilateral e multifocal, com maiores dimensões e apresentando ao diagnóstico invasão da cápsula e metástase ganglionar cervical ou metástase pulmonar $(12,13)$.

\section{ETIOLOGIA}

A tireóide é um dos órgãos mais sensíveis ao efeito tumorigênico das radiações externas, especialmente durante a infância (14), fato bem demonstrado após o acidente nuclear de Chernobyl, na República de Belarus, na Ucrânia, gerando um aumento da incidência de câncer de tireóide nas crianças que atingiu 80 casos anuais por milhão de habitantes $(15,16)$. A sensibilidade da tireóide ao efeito carcinogênico da radiação é máxima durante os primeiros anos de vida e decresce com o aumento da idade no momento da exposição. O risco de tumores não aumenta para exposição após os 15-20 anos de idade (13). Pode haver um período de 5 a 10 anos após a exposição para que os nódulos de tireóide sejam detectados.

O câncer de tireóide também é mais prevalente em regiões carentes de iodo, bem como em associação a doenças tireoideanas autoimunes (17) ou elevação prolongada do TSH (18).

Em cerca de $5 \%$ das crianças há história familiar de PTC. Isto pode ocorrer em famílias com polipose adenomatosa ou doença de Cowden (19).

Em nosso amostra de 20 casos, todos os fatores de risco relacionados ao desenvolvimento do câncer foram investigados, não havendo nenhum paciente com história de irradiação cervical. História de doença tireoideana familiar não-neoplásica foi descrita em 3 casos (12).

\section{GENÉTICA}

Vários defeitos genéticos têm sido demonstrados no PTC da infância, incluindo o rearranjo do RET/PTC e, com menos freqüência, mutação pontual ativadora na via de transdução de sinal dos genes RAS e BRAF (20).

Vários tipos de rearranjo do gene RET têm sido descritos $(21,22)$, com freqüência variável entre os diferentes continentes e com a faixa etária $(23,24)$.

Nos Estados Unidos, cerca de $71 \%$ das crianças têm PTC esporádicos, enquanto 87\% dos PTC de crianças que vivem em áreas contaminadas por irradiação na República de Belarus contêm rearranjos no gene RET $(25,26)$. A maioria dos casos $(n=57)$ era de crianças com PTC induzido por irradiação, onde se encontrou predominância da mutação PTC-3. Em 17 crianças com PTC espontâneo a mutação PTC-1 foi predominante.

A mutação do RET/PTC é encontrada com maior freqüência na criança em relação ao adulto $\mathrm{em}$ casos de PTC espontâneo, mas tanto na criança como no adulto não se correlaciona à extensão da doença no momento do diagnóstico ou à evolução clínica em curto prazo (27).

A mutação BRAFT1796A é a alteração genética mais comum no câncer de tireóide e ocorre exclusivamente no PTC e no carcinoma anaplásico derivado do PTC, especialmente nos subtipos do PTC com células altas e variantes clássicas, os quais são mais associados a invasão extra-tireoideana, metástase linfática e tumores em estágio mais avançado (28). Embora menos freqüente que nos adultos, a mutação BRAFT1796A pode ser encontrada também no PTC da infância, independentemente da exposição à irradiação. A diferença na prevalência dessa mutação entre crianças e adultos portadores de PTC pode estar relacionada à diferença clínica observada entre os dois grupos (29).

\section{APRESENTAÇÃO CLÍNICA}

Em nossa instituição, realizamos análise retrospectiva de 20 pacientes ( 15 meninas e 5 meninos) acompanhados entre 1982 e 2002. A apresentação clínica mais freqüente foi a presença de um nódulo assintomático na 
região cervical anterior presente em 90\% (18/20), nos quais $60 \%$ havia comprometimento concomitante de linfonodos cervicais no momento do diagnóstico (12), não representando este um fator de pior prognóstico (30). Um dos nossos pacientes era portador da doença de Basedow-Graves, tendo sido previamente operado pelo grande volume do bócio. Neste paciente, o exame anátomo-patológico revelou um PTC in situ. Outra criança apresentou quadro inicial de tireoidite aguda com sinais flogísticos locais, e a punção aspirativa por agulha fina (PAAF) mostrou ser um PTC. A presença de um nódulo tireoideano palpável na criança é mais suspeita de ser um câncer que no adulto. A presença de metástase pulmonar é mais freqüente que no adulto, sendo detectada em 31\% dos nossos casos. Convém lembrar que, embora seja um sinal inespecífico, a adenomegalia cervical pode ser o primeiro sinal do câncer de tireóide na infância (12).

A ultra-sonografia é o melhor exame na avaliação do nódulo tireoideano, podendo sugerir, mas não diagnosticar, a etiologia do nódulo. A PAAF é o procedimento mais importante para o diagnóstico etiológico do nódulo de tireóide. De preferência, a PAAF deve ser guiada pelo ultra-som, especialmente em lesões císticas que apresentem vegetações em seu interior (31).

A tabela 1 resume os dados clínicos, laboratoriais e a cintilografia dos pacientes e, na tabela 2, estão descritos os resultados da ultra-sonografia, PAAF e da biópsia cirúrgica.
Os achados patológicos foram analisados de acordo com os parâmetros do TNM. Doze pacientes (63\%) foram classificados com estágio I e 7, com estágio II.

A classificação pelo TNM indica que as crianças apresentam baixo risco de morte relacionada ao câncer, mas subestima o risco de recorrência, que é maior que nos adultos. A morte pelo câncer ocorre na idade adulta, sendo mais freqüente em pacientes cujo câncer apareceu abaixo dos 10 anos $(13,32)$.

\section{TRATAMENTO}

O tratamento é baseado na cirurgia, radioiodoterapia e reposição hormonal, não havendo indicação para radioterapia externa nem quimioterapia.

O tratamento inicial para os cânceres de tireóide é a cirurgia. Como a apresentação do PTC na infância geralmente é multifocal e bilateral, a tireoidectomia total deve ser realizada com a ressecção dos linfonodos acometidos, que são muito freqüentes já ao diagnóstico (33).

A tireoidectomia total com ressecção dos linfonodos foi realizada em 10 (50\%) dos nossos pacientes, tireoidectomia total sem ressecção de linfonodos em 7 (35\%) e hemi-tireoidectomia em um menino de 16 anos com PTC encapsulado de $1 \mathrm{~cm}$ de diâmetro. Este paciente tem segmento de 7 anos sem

Tabela 1. Dados clínicos, laboratoriais, cintilográficos dos pacientes com tumor de tireóide.

\begin{tabular}{lc}
\hline Dados clínicos e laboratoriais & Número de pacientes \\
Sexo & 15 meninas, 5 meninos \\
Bócio uninodular & 12 \\
Bócio multinodular & 6 \\
Bócio difuso & 1 \\
Sem bócio e adenomegalia cervical & 1 \\
Adenomegalia cervical & 11 \\
Disfagia e rouquidão & 2 \\
Massa cervical com sinal flogístico & 1 \\
ATPO e ATg positivos & $2 / 12$ \\
TSH, T4 ou T4 livre anormal & $1 / 12$ \\
Nódulo único e frio & $7 / 12$ \\
Nódulo morno & $2 / 12$ \\
Múltipols nódulos frios & $2 / 12$ \\
Bócio difuso & $1 / 12$ \\
\hline
\end{tabular}

Tabela 2. Dados da ultra-sonografia, PAAF e anátomo-patológico dos pacientes.

\begin{tabular}{lll}
\hline Ultra-som (n= 15) & PAAF (n= 12) & Biópsia cirúrgica (n= 20) \\
Nódulo sólido único (9) & Carcinoma papilífero (5) & Carcinoma papilífero (18) \\
Bócio multinodular (4) & Carcinoma medular (1) & Carcinoma medular (1) \\
Nódulo cístico/sólido (1) & Bócio colóide (1) & Carcinoma folicular (1) \\
Nódulo cístico com vegetação (1) & $\begin{array}{l}\text { Hiperplasia folicular sem atipia (4) } \\
\text { Padrão folicular sem atipia (1) }\end{array}$ & \\
\hline
\end{tabular}


recorrência. Um paciente encaminhado de outro hospital 3 anos após nodulectomia, na idade de 7 anos, desenvolveu metástase pulmonar vindo a falecer 5 anos após a cirurgia, fato que reforça a indicação de tireoidectomia total nas crianças portadoras de câncer de tireóide, em especial abaixo dos 10 anos. Um paciente tinha doença de Graves e o PTC foi encontrado no exame anátomo-patológico da peça cirúrgica, sendo o paciente submetido a tireoidectomia total num segundo tempo.

A metástase pulmonar, muitas vezes já presente no diagnóstico, pode ser do tipo miliar e não visível ao RX ou CT, e somente ser evidenciada pela PCI após a tireoidectomia (13). Trinta por cento dos nossos pacientes apresentavam metástase pulmonar no diagnóstico (12), dados concordantes com Cardoso e cols. (34).

Após a cirurgia, devemos realizar a PCI e administrar dose ablativa de ${ }^{131} \mathrm{I}$, não totalmente estabelecida na população pediátrica. A radioiodoterapia ablativa foi realizada em 18 dos 20 pacientes, com doses variando de 100 a $450 \mathrm{mCi}$, dependendo da presença de metástases. Dois pacientes não realizaram a PCI, um com carcinoma medular e outro submetido à hemi-tireoidectomia.

Em 1992, Samann e cols. (35) avaliaram diferentes protocolos terapêuticos para o carcinoma diferenciado em 140 crianças e adolescentes e sugeriram que a tireoidectomia total, seguida da raidoiodoterapia ablativa, é o tratamento de escolha se a PCI é positiva.

A resposta terapêutica ao ${ }^{131}$ I está relacionada à expressão da proteína NIS na lesão. Em 2002, Patel e cols. (36), utilizando técnica de imuno-histoquímica, demonstrou que a dose de ${ }^{131}$ I necessária para se atingir a remissão era maior nas crianças que expressavam menor quantidade de NIS no tumor, e concluíram que a expressão da NIS está associada a menor risco de recorrência do PTC na criança e no adolescente.

A ablação do tecido tireoideano com 131I aumenta a sensibilidade da medida da tireoglobulina na detecção de recorrência da doença (35).

Após a tireoidectomia, os pacientes devem receber L-tiroxina em dose suficiente para manter o TSH $<0,1 \mathrm{mcU} / \mathrm{mL}$ com o objetivo de suprimir o crescimento tumoral TSH-dependente. Comparado aos adultos, a dose necessária por $\mathrm{kg}$ de peso corporal é geralmente maior. A medida plasmática do TSH e do T4 livre deve ser feita no início a cada 3 meses e posteriormente a cada 6 meses. O crescimento se mantém normal, a puberdade se instala na época adequada e não se descreve efeito colateral sobre o coração ou esqueleto na idade adulta (13).
Na criança e no adolescente, apesar da freqüente detecção de metástases no momento do diagnóstico, o carcinoma diferenciado da tireóide tem prognóstico melhor que no adulto $(37,38)$. Relata-se $90 \%$ de expectativa de vida acima de 15 a 20 anos de seguimento $(19,39)$. Embora o tempo médio de seguimento de nossos casos seja de 9 anos, temos casos com 20 anos de evolução sem sinais de recorrência da doença.

\section{DIAGNÓSTICO E TRATAMENTO DO CÂNCER PERSISTENTE OU RECORRENTE}

A procura pela doença persistente deve ser realizada pela PCI 3 a 5 dias após a administração da dose ablativa do 131I. Qualquer foco de captação fora do leito tireoideano deve ser considerado disseminação metastática. A medida da tireoglobulina $(\mathrm{Tg})$ sérica é um marcador valioso da presença de metástase. Inicialmente deve ser determinada com o TSH desbloqueado ( $\mathrm{TSH}>30 \mathrm{mcU} / \mathrm{mL}$ ) ou sob estímulo com o TSH recombinante (40). A retirada prolongada da tiroxina com a finalidade de aumentar o TSH endógeno também é mal tolerada pelas crianças. Quando não se detecta alteração da Tg medida sob estímulo, a probabilidade de doença persistente ou recorrência é muito pequena. Os cuidados com o resultado falso-positivo associado à presença de anticorpos anti-tireoglobulina também devem ser observados.

Como a sensibilidade palpatória na detecção de linfoadenomegalia é baixa, recomenda-se a realização de ultra-sonografia cervical periódica para essa finalidade. A detecção de um nódulo aumentado deve indicar uma PAAF para exame citológico e medida da Tg no líquido aspirado.

O tratamento das metástases pulmonar é realizado com ${ }^{131}$ I. Nesses casos, freqüentemente encontramos linfoadenomegalia mediastinal, que pode requerer tratamento cirúrgico. A velocidade de crescimento das metástases é muito pequena sob a terapia supressiva do TSH, mas, como a expectativa de vida é longa, a presença de doença persistente pode causar a morte décadas após o tratamento inicial.

A ocorrência de um segundo câncer não deve ser esquecida, especialmente na criança com câncer de tireóide.

\section{CONCLUSÃO}

Na infância e na adolescência, todo nódulo tireoideano persistente deve ser cuidadosamente avaliado pelo risco de se tratar de um carcinoma. A ultra-sonografia e a 
PAAF são os exames mais importante na elucidação do diagnóstico. O tratamento do câncer diferenciado de tireóide na infância e na adolescência deve ser a tireoidectomia total com esvaziamento ganglionar regional. Após a cirurgia, devemos realizar a PCI e a radioiodoterapia. A medida da Tg plasmática sob estímulo do TSH é um bom parâmetro de cura. A terapia de reposição com L-tiroxina deve utilizar dose suficiente para manter o $\mathrm{TSH}<0,1 \mathrm{mcU} / \mathrm{mL}$. Com tratamento adequado, o prognóstico é melhor que nos adultos.

\section{AGRADECIMENTOS}

Agradecemos ao Núcleo de Apoio à Publicação da Faculdade de Ciências Médicas da Santa Casa de São Paulo - NAP-SC pelo o suporte técnico-científico à publicação deste manuscrito.

\section{REFERÊNCIAS}

1. Rallison ML, Dobyns BM, Keating FR Jr, Rall JE, Tyler FH. Thyroid nodularity in children. JAMA 1975;233:1069-72.

2. Buckwalter JA, Gurll NJ, Thomas CG Jr. Cancer of the thyroid in youth. World J Surg 1981;5:15-25.

3. Hung W, Sarlis NJ. Current controversies in the management of pediatric patients with well-differentiated nonmedullary thyroid cancer: a review. Thyroid 2002;12:683-702.

4. Schlumberger MJ. Papillary and follicular thyroid carcinoma. N Engl J Med 1998;338:297-306.

5. Kirkland RT, Kirkland JL, Rosenberg HS, Harberg FJ, Librik L, Clayton GW. Solitary thyroid nodules in 30 children and report of a child with a thyroid abscess. Pediatrics 1973;51:85-90.

6. Siguero JPL, Molina JAM, García MA Cáncer de tiroides en la infancia. In: Arias MP (ed). Tratado de Endocrinología Pediátrica. 2a ed. Madrid: Diaz de Santos, 1997. pp. 609-20.

7. Greenlee RT, Hill-Harmon MB, Murray T, Thun M. Cancer statistics, 2001. CA Cancer J Clin 2001;51:15-36.

8. Foley Jr TP. Disorders of the thyroid in children. In: Sperling $M$ (ed). Pediatric Endocrinology. Philadelphia: WB Saunders Co, 1996. pp. 171-94.

9. Franceschi $S$, Boyle $P$, Maisonneuve $P$, La Vecchia C, Burt AD, Kerr DJ, et al. The epidemiology of thyroid carcinoma. Crit Rev Oncog 1993:4:25-52.

10. Millman B, Pellitteri PK. Thyroid carcinoma in children and adolescents. Arch Otolaryngol Head Neck Surg 1995; $121: 1261-4$

11. Hung W. Well-differentiated thyroid carcinomas in children and adolescents: a review. Endocrinologist 1994;4:117-25.

12. Monte O, Longui CA, Calliari LE, Kochi C, Scalissi NM, Uvo RA, et al. Thyroid carcinoma during childhood and adolescence. Endocrinologist 2005; 15:106-10

13. Leboulleux S, Baudin E, Hart DW, Travagli JP, Schlumberg M. Follicular cell-derived thyroid cancer in children. Horm Res 2005:63:145-51.

14. Ron E, Lubin JH, Shore RE, Mabuchi K, Modan B, Pottern LM, et al. Thyroid cancer after exposure to external radiation: a pooled analysis of seven studies. Radiat Res 1995; 141:259-77.

15. Reiners C, Biko J, Demidchik YE, Drozd VM. Results of radioiodine treatment in children from Belarus with advanced stages of thyroid cancer after the Chernobyl accident. In: 'Chernobyl: Message for the 21st Century'. Excerpta Medica International Congress series 1234. Amsterdam: Elsevier, 2002. pp. 69-75.
16. Kazakov VS, Demidchik EP, Astakhova LN. Thyroid cancer after Chernobyl. Nature 1992;359:21-5.

17. Kamma H, Fujii K, Ogata T. Lymphocytic infiltration in juvenile thyroid carcinoma. Cancer 1988;62:1988-93.

18. Feinmesser R, Lubin E, Segal K, Noyek A. Carcinoma of the thyroid in children - a review. J Pediatr Endocrinol Metab 1997; 10:561-8.

19. Malchoff CD, Malchoff DM. The genetics of hereditary nonmedullary thyroid carcinoma. J Clin Endocrinol Metab 2002;87:2455-9.

20. Nikiforova MN, Kimura ET, Gandhi M, Biddinger PW, Knauf $\mathrm{JA}$, Basolo $\mathrm{F}$, et al. BRAF mutations in thyroid tumors are restricted to papillary carcinomas and anaplastic or poorly differentiated carcinomas arising from papillary carcinomas. J Clin Endocrinol Metab 2003;88:5399-404.

21. Klugbauer S, Rabes HM. The transcription coactivator HTIF1 and a related protein are fused to the RET receptor tyrosine kinase in childhood papillary thyroid carcinomas. Oncogene 1999:18:4388-93.

22. Nakata T, Kitamura $Y$, Shimuzu K, Tanaka S, Fujimori M, Yokoyama $S$, et al. Fusion of a novel gene, ELKS, to RET due to translocation $\mathrm{t}(10 ; 12)(\mathrm{q} 11 ; \mathrm{p} 13)$ in a papillary thyroid carcinoma. Genes Chromosomes.Cancer 1999:25:97-103.

23. Santoro M, Carlomagno F, Melillo RM, Billaud M, Vecchio G, Fusco A. Molecular mechanisms of RET activation in human neoplasia. J Endocrinol Invest 1999:22:811-9.

24. Pacini F, Elisei R, Romei C, Pinchera A. RET proto-oncogene mutations in thyroid carcinomas: clinical relevance. $\mathbf{J}$ Endocrinol Invest 2000;23:328-38.

25. Motomura T, Nikiforov YE, Namba H, Ashizawa K, Nagataki S, Yamashita $S$, et al. ret rearrangements in Japanese pediatric and adult papillary thyroid cancers. Thyroid 1998;8:485-9.

26. Nikiforov YE, Rowland JM, Bove KE, Monforte-Munoz $\mathrm{H}$, Fagin JA. Distinct pattern of ret oncogene rearrangements in morphological variants of radiation-induced and sporadic thyroid papillary carcinomas in children. Cancer Res 1997; 57:1690-4.

27. Fenton $C L$, Lukes $Y$, Nicholson $D$, Dinauer $C A$, Francis $G L$, Tuttle RM. The ret/PTC mutations are common in sporadic papillary thyroid carcinoma of children and young adults. $\mathbf{J}$ Clin Endocrinol Metab 2000;85:1170-5.

28. Xing $M$, Westra WH, Tufano RP, Cohen $Y$, Rosenbaum E, Rhoden $\mathrm{KJ}$, et al. BRAF mutation predicts a poorer clinical prognosis for papillary thyroid cancer. J Clin Endocrinol Metab 2005;90:6373-9.

29. Kumagai $A$, Namba $H$, Saenko VA, Ashizawa K, Ohtsuru A, Ito $\mathrm{M}$, et al. Low frequency of BRAFT1796A mutations in childhood thyroid carcinomas. J Clin Endocrinol Metab 2004; $89: 4280-4$.

30. Gorlin JB, Sallan SE. Thyroid cancer in childhood. Endocrinol Metab Clin North Am 1990;19:649-62.

31. Tomimori EK, Camargo RY, Bisi H, Medeiros-Neto G. Combined ultrasonographic and cytological studies in the diagnosis of thyroid nodules. Biochimie 1999;81:447-52.

32. Schlumberger $M$, De Vathaire $F$, Travagli JP, Vassal G, Lemerle J, Parmentier C, et al. Differentiated thyroid carcinoma in childhood: long term follow-up of 72 patients. J Clin Endocrinol Metab 1987;65:1088-94.

33. Haveman JW, van Tol KM, Rouwe CW, Piers DA, Plukker JT. Surgical experience in children with differentiated thyroid carcinoma. Ann Surg Oncol 2003;10:15-20.

34. Cardoso AA, Pianovski MAD, França SN, Pereira RM, Boguzewski M, Sandrini R, et al. Câncer de tireóide na infância e adolescência - Relato de 15 Casos. Arq Bras Endocrinol Metab 2004;48:835-41.

35. Samaan NA, Schultz PN, Hickey RC, Goepfert H, Haynie TP, Johnston DA, et al. The results of various modalities of treatment of well differentiated thyroid carcinomas: a retrospective review of 1,599 patients. J Clin Endocrinol Metab 1992; 75:714-20.

36. Patel A, Jhiang S, Dogra S, Terrell R, Powers PA, Fenton C, et al. Differentiated thyroid carcinoma that express sodiumiodide symporter have a lower risk of recurrence for children and adolescents. Pediatr Res 2002;52:737-44. 
37. Zimmerman D, Hay ID, Gough IR, Goellner JR, Ryan JJ, Grant CS, et al. Papillary thyroid carcinoma in children and adults: long-term follow-up of 1,039 patients conservatively treated at one institution during three decades. Surgery 1988; $104: 1157-66$

38. Vassilopoulou-Sellin R, Libshitz HI, Haynie TP. Papillary thyroid cancer with pulmonary metastases beginning in childhood: clinical course over three decades. Med Pediatr Oncol 1995;24:119-22.

39. Mazzaferri EL. An overview of the management of papillary and follicular thyroid carcinoma. Thyroid 1999;9:421-7.

40. Iorcansky S, Herzovich V, Qualey RR, Tuttle RM. Serum thyrotropin (TSH) levels after recombinant human TSH injections in children and teenagers with papillary thyroid cancer. J Clin Endocrinol Metab 2005;90:6553-5.
Endereço para correspondência:

Osmar Monte

Rua Ceará 247

01243-010 São Paulo, SP

Fax: (1 1) 3663-6333

E-mail: dir.fisio@santacasasp.org.br / fcmnapsc@santacasasp.org.br 